\title{
Can Machine Translation Assist to Prepare for Simultaneous Interpretation?
}

\author{
https://doi.org/10.3991/ijet.v15i16.14935 \\ Ying Qin ${ }^{(凶)}$, Chunqing Wang \\ Beijing Foreign Studies University, Beijing, China \\ qinying@bfsu.edu.cn
}

\begin{abstract}
With the rapid development of machine translation, the research of man-machine coordinated translation is drawing much concern. This paper explores roles of machine translation on advance preparation for simultaneous interpreting. We carry out a comparative study on 14 student interpreters, which are divided into experimental group and control group. The students in experimental group are allowed to use machine translation to prepare for a simultaneous interpreting, while the students in control group are not. The experimental results show that errors in machine translation might mislead the students in the simultaneous interpreting practice. On the other hand, if the time of preparation is limited, it is better not to resort to machine to make preparation because of time-consuming proofreading, or twice the effort would achieve half the result.
\end{abstract}

Keywords - Simultaneous interpreting, advance preparation for interpretation, machine translation, student interpreters.

\section{Introduction}

With the quality improving of Machine Translation (MT) [1], automatic translation has brought much influence on professional translators though MT still cannot replace human translation at present. Most translators especially the younger generation show open mind to MT and are willing to utilize MT in their daily work. Therefore, the computer-aid translation teaching is gradually adopted in colleges [2]. How MT systems cooperate or coordinate with human translators is under extensive investigation. Several working patterns like aid-translating [3] and post-editing [4] have been proposed. This paper carries out an experiment to explore the effects of MT on the student interpreters who make pre-preparation for conference simultaneous interpretation.

Simultaneous interpretation is a challenge job that the interpreter listens to the speaker's statements in the source language through a headset in a conference and instantaneously translates them into the target language. To carry out simultaneous interpretation task successfully, interpreters usually spend a lot of time preparing for the translation task before the conference starts. There is widespread consensus that interpreters work better when they prepare in advance [5]. Ref [6] sums up seven 
contents of advance preparation including (1) inquiring about the background of the conference, audiences and speakers; (2) asking for the schedule of the conference, the presentation if permitted, or materials of previous conferences; (3) reading through all the materials related to the conference and translating the domain terms; (4) preparing the draft translation of related materials; (5) consulting about the resumes of speakers and their previous speeches; (6) reviewing of glossary and expressions anticipated; (7) checking the venue and devices in advance. Among these works, document translation related to the background of the conference such as proper nouns and terminologies is the most important.

There are lots of methods can help the interpreter to fulfill the preparation. So, can MT assist the interpreter to prepare for the simultaneous interpreting?

In the following, we first introduce the setting and procedure of the experiment according to our research purposes. Then we will report and analyze the results in the following. To better know the attitude towards MT, we carry out a post-experiment interview of the participants in section 4 . The last section is the conclusion.

\section{Experiment Settings and Procedure}

Intuitively MT can raise the efficiency and accuracy in advance preparation for simultaneous interpreting, which will result in better quality of interpretation in practice. We then design a comparative experiment to verify whether MT plays the hypothetical role.

With respect to the subjects, we select 14 student interpreters who major in MTI (Master of Translation and Interpretation) and SI (Simultaneous Interpretation) to conduct the experiment. According their usual performances, the students have similar interpreting competence. They are randomly divided into two groups, 7 into the experimental group, while another 7 into the control group. Interpreters in experimental group is required to use machine translation in the preparation before interpretation, while the students in control group are not.

All the participants are informed about the topic of the conference and background of the speaker in advance. They can search for the related materials according to individual anticipation. To test the efficiency of using MT, we limit the time of preparation according to the length and difficulty of the presentation. Experimental group is allowed to use any online MT system to help translate the presentation, while the control group is not allowed but can use the search engine and other resources. When the time due, the voice record of the conference is played to simulate the actual scene and the simultaneous interpreting set off. All the performances of interpreters are recorded for later transcription and analysis. The final step is the evaluation and comparison of the interpretation performance of the two groups. After the experiment, we further interview the student interpreters in the experimental group and know more about their attitudes towards machine translation.

The translation direction is English into Chinese, which the target language is the mother tongue of the student interpreters. 
We also select the experimental corpus considering the degree of difficulty and normality. The conference record is the speech on the topic of economic reform in length of 12.31 minutes, without accent. The transcription of speech consists of 67 sentences, totally 1459 words. The average length of sentence is 21.8 , with the range from 3 to 76. There are 11 sentences containing more than 30 words. And the degree of translation difficulty is moderate for these students. Each interpreter is given thirty minutes to prepare before the conference starts. Thirty minutes is a little urgent for the students, since we want to check whether machine translation can dramatically reduce the time of preparation.

\section{Experiment Results and Analysis}

Quality of interpretation is fundamental. Twenty-four errors are made in the experimental groups while only 6 errors in the control group, though the interpreters in both groups have similar competence according to their previous performances. We suspect the significant difference resulting from interference of machine translation. Therefore, we focus on the translation errors in the presentation as they use machine translation to prepare for the conference.

\subsection{Influence from machine translation errors}

Four types of MT error are identified including (1) core meaning divergence, (2) moderate semantic error, (3) improper technical terms or proper nouns, and (4) minor mistakes like non-standard unit of measurement and abbreviation. The degree of severity is listed in descending order.

With a thorough check, there are 8 core meaning divergences, 3 moderate semantic errors and 1 improper terminology in the machine translation of the presentation. We then compare the interpretation mistakes made by each student in experiment group and control group separately.

Table 1 shows the performance of students in experimental group when encountering the related sentences containing core meaning translation errors. The comparative performance of control group on these sentences are also shown in the table. The digit represents the number of interpreters.

Table 1. Influence of core meaning errors in MT

\begin{tabular}{|c|c|c|c|c|}
\hline & \multicolumn{2}{|c|}{ Experimental group } & \multicolumn{2}{c|}{ Control group } \\
\hline Sentence & correct & wrong & correct & wrong \\
\hline S1 & 4 & 3 & 6 & 1 \\
\hline S2 & 0 & 7 & 5 & 2 \\
\hline S3 & 5 & 2 & 7 & 0 \\
\hline S4 & 6 & 1 & 6 & 1 \\
\hline S5 & 4 & 3 & 7 & 0 \\
\hline S6 & 7 & 0 & 7 & 0 \\
\hline S7 & 5 & 2 & 6 & 0.75 \\
\hline S8 & 3 & 4 & 6.25 & \\
\hline Average & 4.25 & 2.75 & & \\
\hline
\end{tabular}


Table 1 obviously tells us that severe errors made by MT interfere with the interpretation of students in experimental group. The wrong translations by machine in the preparation stage probably induce the mistakes of the students. Detailed analysis is in the next subsection.

With respect to the moderate semantic errors and improper technical terms, their influences are less than the core errors as shown in Table 2.

Table 2. Influence of moderate semantic errors and technical terms in MT

\begin{tabular}{|c|c|c|c|c|}
\hline & \multicolumn{2}{|c|}{ Experimental group } & \multicolumn{2}{c|}{ Control group } \\
\hline Sentence & correct & wrong & correct & wrong \\
\hline S1 & 7 & 0 & 7 & 0 \\
\hline S2 & 6 & 1 & 7 & 0 \\
\hline S3 & 7 & 0 & 7 & 0 \\
\hline S4 & 6 & 1 & 7 & 0 \\
\hline Average & 6.5 & 0.5 & 7 & 0 \\
\hline
\end{tabular}

We further check whether these mistakes are centered on some students, that is, the interfere from MT is limited. Fig. 1 shows the error distribution among the seven interpreters, in which Ix denotes the interpreter in the experiment group.

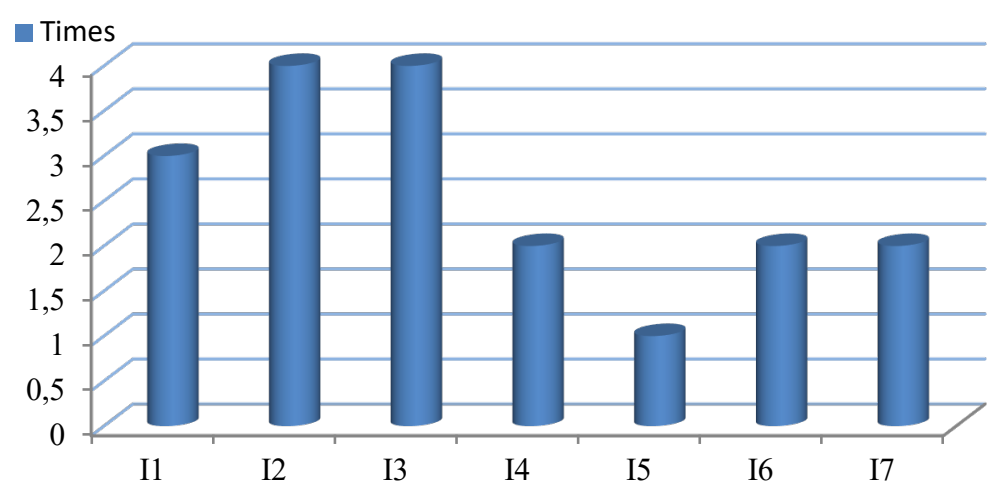

Fig. 1. Interference distribution among interpreters

According to Fig. 1, each interpreter is interfered at least once. The average inference from MT error is 2.6. The phenomenon indicates the students rely on the machine translation to prepare for the simultaneous interpretation. Generally they trust the result of machine translation and do not make further verification.

We also calculate all the mistakes made by each student and check whether it is affected by the wrong result of machine translation, as shown in Table 3 . 
Table 3. Mistakes made by each interpreter

\begin{tabular}{|c|c|c|}
\hline Sentence & Total number & Interfered number \\
\hline S1 & 3 & 3 \\
\hline S2 & 7 & 7 \\
\hline S3 & 2 & 2 \\
\hline S4 & 1 & 1 \\
\hline S5 & 3 & 2 \\
\hline S6 & 0 & 0 \\
\hline S7 & 2 & 0 \\
\hline S8 & 3 & 3 \\
\hline S9 & 0 & 0 \\
\hline S10 & 1 & 0 \\
\hline S11 & 0 & 0 \\
\hline $\mathrm{S} 12$ & 0 & 0 \\
\hline S13 & 1 & 0 \\
\hline
\end{tabular}

The data in Table 3 confirm that most mistakes made by the students are related to the misleading from machine translation. On the contrary, the students of control group rarely make such mistakes in their simultaneous interpretation practice.

\subsection{Detailed analysis}

Here we show two examples to illustrate the influence of MT. Some words in the examples are underlined to indicate they are problematic, hereinafter.

Example (1).

SRC: It has been a lot of topics of interest, especially regarding to the reform that had laid out after the 3rd Plenum decisions.

MT : 这是一个很有意思的话题, 特别是矢于在三中全会决定之后制定的改 革。

All the seven interpreters in experimental group wrongly translate the phrase underlined into 有意思的话题 or 有趣的话题, similar to the machine translation. On the contrary, only one student in control group makes such mistake, others correctly interpret it into 我们共同关切的话题 or 我们感兴趣的话题. The superficial translation from machine misleads the interpreters, ignoring the context of speech.

Example (2).

SRC: So when we compare the spikes, the economic density of Beijing or Shanghai, versus Tokyo, London, Paris 20 years ago, we found that the economic density, we can think of it visually as the height of the spike, for the metropolises in China are way much lower than global cities even 25 years ago.

MT: 因此, 当我们比较20年前北京或上海的经济密度与东京，伦敦，巴黎的 经济密度时, 我们发现中国大都市的经济密度，我们可以将其视为穗的高度。 在25年前，它远远低于全球城市。

This is a difficult speech segment with complex syntactic structure. There are three students in experimental group make mistakes, while only one in control group does. Machine translation of this sentence is an incorrect comparison among the economic 
density of these cities. And three students have similar wrong interpretation as the machine does.

\subsection{Quality Score of interpretation}

According to the evaluation standard [7], simultaneous interpretation quality consists of six aspects including accuracy $(30 \%)$, coherence $(25 \%)$, skill( $15 \%$ ), vocabulary $(10 \%)$, pronunciation $(10 \%)$ and grammaticality $(10 \%)$. We evaluate the students' interpretation using the grading tool based on the standard. Table 4 shows the score of each student's performance.

Table 4. Score of interpretation quality

\begin{tabular}{|c|c|c|}
\hline Interpreter & Experimental group & Control group \\
\hline I1 & 82.6 & 85.05 \\
\hline I2 & 80.9 & 83.75 \\
\hline I3 & 77.15 & 84.75 \\
\hline I4 & 84.9 & 85.3 \\
\hline I5 & 84.95 & 84.05 \\
\hline I6 & 85.15 & 84.8 \\
\hline I7 & 83.4 & 87.15 \\
\hline Average & 82.72 & 84.98 \\
\hline
\end{tabular}

The average score of experimental groups is lower than the control group, which indicates that using MT to prepare for the conference does not benefit the students.

\subsection{Time of preparation}

Three students in experimental group cannot finish preparing in 30 minutes, while all the students in control group finish it in time. Both groups feel it urgent to fulfill the task. Four students complain that they spend a lot of time on the proofreading of machine translation and have no time to search for enough knowledge resources. There are seven kinds of problems in MT which they spend time to correct.

- Intelligible characters in the machine translation

- Uncertain translation of low-frequency words and phrases

- Superficial translation of the collocation and idioms

- Stereotyped translation of the same words without concerning the contexts

- Confused order of sub-sentences caused by wrong structure analysis of long sentences

- Wrong translation of modifiers and parenthesis

- Confused logistic relation among sub-sentences

The students have to modify the errors before referring to the results of machine. When the task is urgent, it is better not to use it. However, it is admitted that the advantage of machine translation is speedy. 


\section{Post-Experiment Interview}

After the experiment, we conduct an interview with the students in the experimental group and know more about their viewpoints.

Although most student interpreters prefer to use MT in their preparation for simultaneous interpreting, the degree of trust on MT is rather low among the students. They are not fully satisfied with the current MT systems. Most of the time they treat it as a bilingual dictionary to translate words or phrases, especially some proper nouns or terminologies. They seldom use MT to translate whole sentences, least of complicated sentences. Hardly they paste the full document to transform.

Among the students who are willing to use MT to prepare, $44 \%$ of them don't care about domains. Others show preferences. The preferable fields in descending order are science and technology (54.67\%), law (45.33\%), finance (42.67\%), economic (30.67\%), agriculture (26.67\%), politics (25.33\%), environment (18.67\%) and arts (4\%). Most students will seek for help of machine when the task has much to do with domain knowledge. As to the humanities and literature, they are hesitating to use MT. A small percentage of students think MT provide them more ideas on translation.

\section{Conclusion}

With MT on hand, student interpreters might refer to the translation of machine when they prepare for a simultaneous interpreting. But the errors in MT would mislead the interpreters, especially students. The experimental results show that when the material is moderately difficult and preparation time is limited, machine translation cannot save the time of advance preparation and cannot improve their interpretation performance either.

This study is limited in the number of sample interpreters and domain coverage of experimental data. We will widen the samples and domains in the future work.

\section{Acknowledgement}

The research is supported by Projects of the National Social Science Foundation of China (No. 17BYY047).

\section{$7 \quad$ References}

[1] Li Huiyan (2019) A novel machine translation method based on stochastic finite automata model for spoken English. International Journal of Emerging Technologies in Learning, v14, n 6, p98-109.

[2] Zhang Wenming, Zhang erwn (2019) Application of neural network machine translation in college translation teaching.International Journal of Emerging Technologies in Learning, v14, n19, p16-25. https://doi.org/10.3991/ijet.v14i19.10690 
[3] Yao Shuping (2017). Application of computer-aided translation in English teaching. International Journal of Emerging Technologies in Learning, v12, n8, p105-117

[4] Sharon Obrien et al. (2014). Post-editing of machine translation: Processes and applications. Cambridge Scholars Publishing.

[5] Stephanie Díaz-Galaz, Presentacion Padilla, M. Teresa Bajo (2015). The role of advance preparation in simultaneous interpreting Interpreting Vol. 17:1, p1-25. https://doi.org/10.1075/intp.17.1.01dia

[6] Zhang Jiliang (2003). Preparation in Interpreting. Chinese Science \& Technology Translators Journal (3): p13-17.

[7] Robin Setton and Andrew Dawrant (2016). Conference Interpreting: A Trainer's Guide. John Benjamins Publishing Company. https://doi.org/10. 7202/1055156ar

\section{Authors}

Ying Qin is a researcher of Artificial Intelligence and Human Languages Lab, Beijing Foreign Studies University. She obtained Ph.D in 2008 and now is an associate professor at School of Information Science and Technology. Her research area is Computational Linguistics including MT, dialogue system and quality evaluation. Details of publishing can be found on her account of Google Scholar, see: https://scholar.google.com/citations?hl=en\&user=Cum7cAkAAAAJ\&view op=list works\&gmla=AJsN-F4EivnaStOqiiI013Cu9yzzs5lWlEpty12-20fpqdV05CzqhlLPmp8 C8t_mieh6XChu3Th6ufCCl5oS-XM5s6Q4DF6DZH2 J33EQqCtWq14NVj-7oYFl7 w-HLa09FQXj-TTQY5q

Chunqing Wang is a master student of Graduate School of Translation and Interpretation, Beijing Foreign Studies University. Her major is Interpretation and Technology.

Article submitted 2020-04-17. Resubmitted 2020-05-22. Final acceptance 2020-05-22. Final version published as submitted by the authors. 